03,06

\title{
Параметрический резонанс и фотогальванические токи в слоистом кристалле $\mathrm{TIGaSe}_{2}$
}

\author{
(C) А.П. Одринский ${ }^{1}$, М.-H.Yu. Seyidov ${ }^{2,3}$, Т.Г. Мамедов ${ }^{2}$, В.Б. Алиева ${ }^{2}$ \\ ${ }^{1}$ Институт технической акустики НАН Беларуси, \\ Витебск, Беларусь \\ ${ }^{2}$ Институт фризики НАН Азербайджана, \\ Баку, Азербайджан \\ ${ }^{3}$ Department of Physics, Gebze Technical University, \\ Gebze, Kocaeli, Turkey \\ E-mail: odra@mail333.com
}

(Поступила в Редакцию 18 мая 2016 г.)

\begin{abstract}
Представлены результаты феноменологического исследования кинетики аномального фотоотклика слоистого сегнетоэлектрика-полупроводника $\mathrm{TlGaSe}_{2}$ в области температур $T \sim 170-280 \mathrm{~K}$, соответствующей параэлектрической фазе кристалла. С учетом изменений с температурой кинетики фотоотклика предложены основные механизмы возникновения аномалий, связанные с пространственной неоднородностью локализованных и нелокализованных зарядов в объеме кристалла. Предложен механизм возникновения параметрического резонанса как обусловленного фотогальваническими токами в кристалле.
\end{abstract}

DOI: 10.21883/FTT.2017.03.44152.189

\section{1. Введение}

Представители семейства тройных халькогенидных соединений $\mathrm{A}^{\mathrm{III}} \mathrm{B}^{\mathrm{III}} \mathrm{C}_{2}^{\mathrm{IV}}-$ слоистые монокристаллы сегнетоэлектрика-полупроводника $\mathrm{TlGaSe}_{2}$ - привлекают внимание исследователей рядом уникальных свойств, интересных в связи с практическим применением [1]. Согласно данным [2,3], ширина запрещенной зоны этих кристаллов, определяемая при $300 \mathrm{~K}$, варьируется в пределах $1.83-2.13 \mathrm{eV}$ для прямых оптических переходов и $2.00-2.23 \mathrm{eV}$ для непрямых оптических переходов. С понижением температуры в $\mathrm{TlGaSe}_{2}$ наблюдается последовательность фазовых переходов из высокотемпературной параэлектрической фазы в несоразмерную фазу (вблизи $T \sim 120 \mathrm{~K})$ и далее $(T \sim 110 \mathrm{~K})$ из несоразмерной в соразмерную сегнетоэлектрическую фазу. Однако ввиду высокого удельного сопротивления $\mathrm{TlGaSe}_{2}$ и в параэлектрической фазе обнаруживает склонность к формированию электрических неоднородностей в объеме кристалла [4-6], а также проявлению иных аномалий: резонанса полярных колебаний кристаллической решетки типа мягкой моды [7], изменения характера температурной зависимости и структуры спектров ядерного магнитного резонанса $[8,9]$, скачкообразного изменения фототока [10], особенностей на температурной зависимости теплоемкости $[11,12]$ и коэффициентов теплового расширения кристалла [13]. Электрически активные дефекты слоистого кристалла сегнетоэлектрика-полупроводника $\mathrm{TlGaSe}_{2}$, способные к локализации носителей заряда и как следствие ответственные за формирование электрических неоднородностей в объеме кристалла, исследованы в [4-6]. В процессе отработки режимов регистрации кинетики релаксации, оптимальных для обнаружения термоэмиссии неравновесного заполнения дефектов, в соответствии с методиками фотоэлектрической релаксационной спектроскопии (PICTS) [14] в области температур параэлектрического состояния кристалла были выявлены условия регистрации кинетики фотоотклика кристалла $\mathrm{TlGaSe}_{2}$ аномального типа. Pезультаты исследования аномального фотоотклика представлены в настоящей работе.

\section{2. Описание эксперимента}

Выращенные методом Бриджмена-Стокбаргера, специально не легированные монокристаллы $\mathrm{TlGaSe}_{2}$ из разных технологических партий обладали проводимостью $p$-типа. Удельное сопротивление образца G130 составляло $1.3 \cdot 10^{6} \Omega \cdot \mathrm{cm}$, а образца Ga3 $2.8 \cdot 10^{6} \Omega \cdot \mathrm{cm}$. Образцы имели размер $2 \times 4 \times 0.3 \mathrm{~mm}$. Омические контакты формировались заливкой торцевых поверхностей образца серебряной пастой. Качество контактов оценивалось по вольт-амперной характеристике, которая была линейной при комнатной температуре. Геометрия протекания тока - вдоль слоев кристалла. Световое возбуждение проводилось перпендикулярно поверхности кристалла - плоскости скола - и соответствовало плотности потока фотонов на поверхности кристалла $\sim 10^{14} \mathrm{~cm}^{-2} \cdot \mathrm{s}^{-1}$. Использовалось квазимонохроматическое возбуждение с энергией фотонов $h v=2.02 \mathrm{eV}$ при $\Delta h v=0.11 \mathrm{eV}$ (излучение ксеноновой лампы ДКСШ-500, прошедшее через монохроматор типа МСД-1 при ширине входной и выходной щелей $4 \mathrm{~mm}$ ). Наложение от других порядков дифракционной решетки убиралось соответствующими светофильтрами. Периодичность возбуждения составляла $\sim 300 \mathrm{~ms}$ при длительности светового периода $\sim 50 \mathrm{~ms}$. 
Методика измерений не отличалась от описанной в [5]. Регистрация данных проводилась в процессе нагрева образца со скоростью $\sim 2 \mathrm{~K} / \mathrm{min}$ в диапазоне температур $78-330 \mathrm{~K}$ с шагом $1 \mathrm{~K}$. При регистрации кинетики релаксации фотоотклика проводились поточечное накопление и усреднение кинетики сигнала (64 реализации), содержащей 2000 отсчетов с интервалом дискретизации по времени $\tau=6.3 \cdot 10^{-5} \mathrm{~s}$. Параллельно по аналогичной методике регистрировалась кинетика самого фотоотклика ( 10 реализаций), содержащая 500 отсчетов с интервалом дискретизации по времени $6 \tau$.

\section{3. Феноменологическое описание}

Аномалии кинетики фотоотклика наблюдались в области температур $170-300 \mathrm{~K}$ в условиях периодического светового возбуждения с энергией фотонов, превышающей или близкой к ширине запрещенной зоны кристалла $\left(h v_{0} \geq E_{g}\right)$. Образцы фотоотклика с явно выраженным немонотонным характером реакции на возбуждение светом и релаксации фотоотклика представлены на рис. 1 . Кривая 1 на рис. $1, a$, полученная при температу-
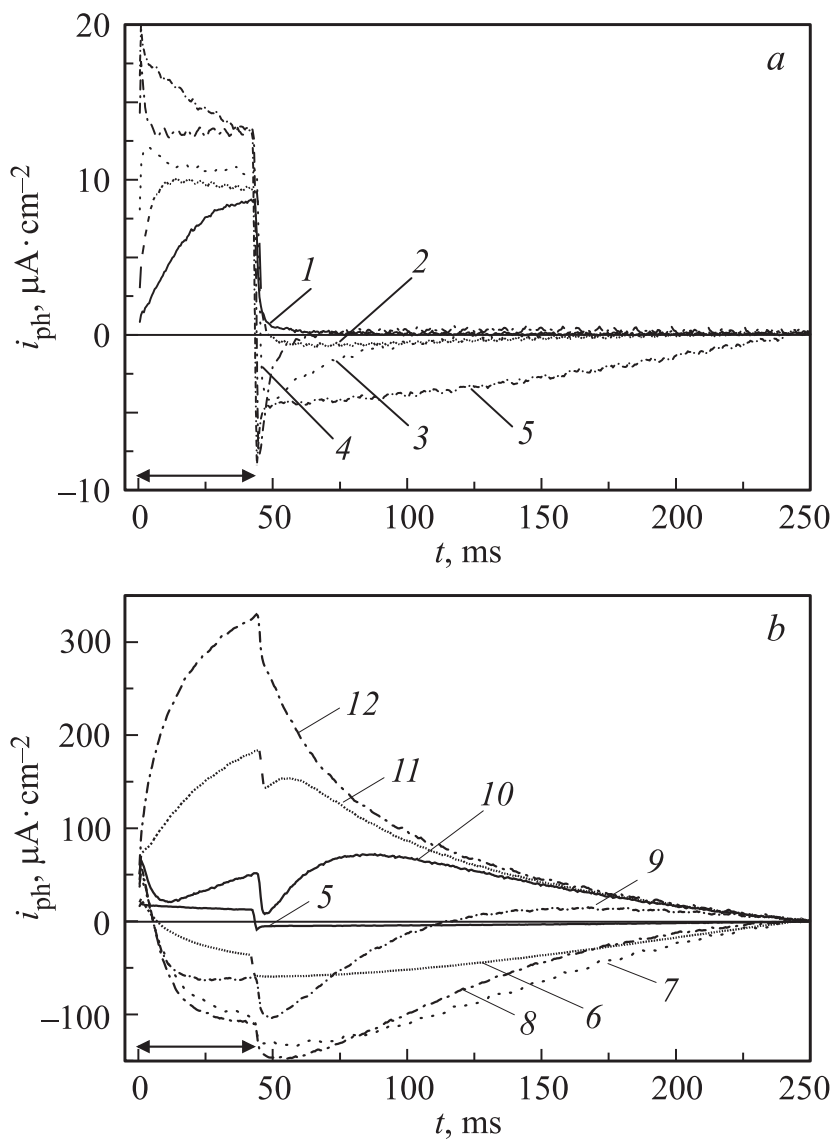

Рис. 1. Кинетика фотоотклика образца $\mathrm{Ga} 3$ в области температур $T=170-210(a)$ и $210-280 \mathrm{~K}(b) . T=170(1), 180(2)$, 190 (3), 200 (4), 210 (5), 220 (6), 230 (7), 240 (8), 250 (9), $260(10), 270(11)$ и $280 \mathrm{~K}(12)$. Стрелкой отмечен период светового возбуждения.
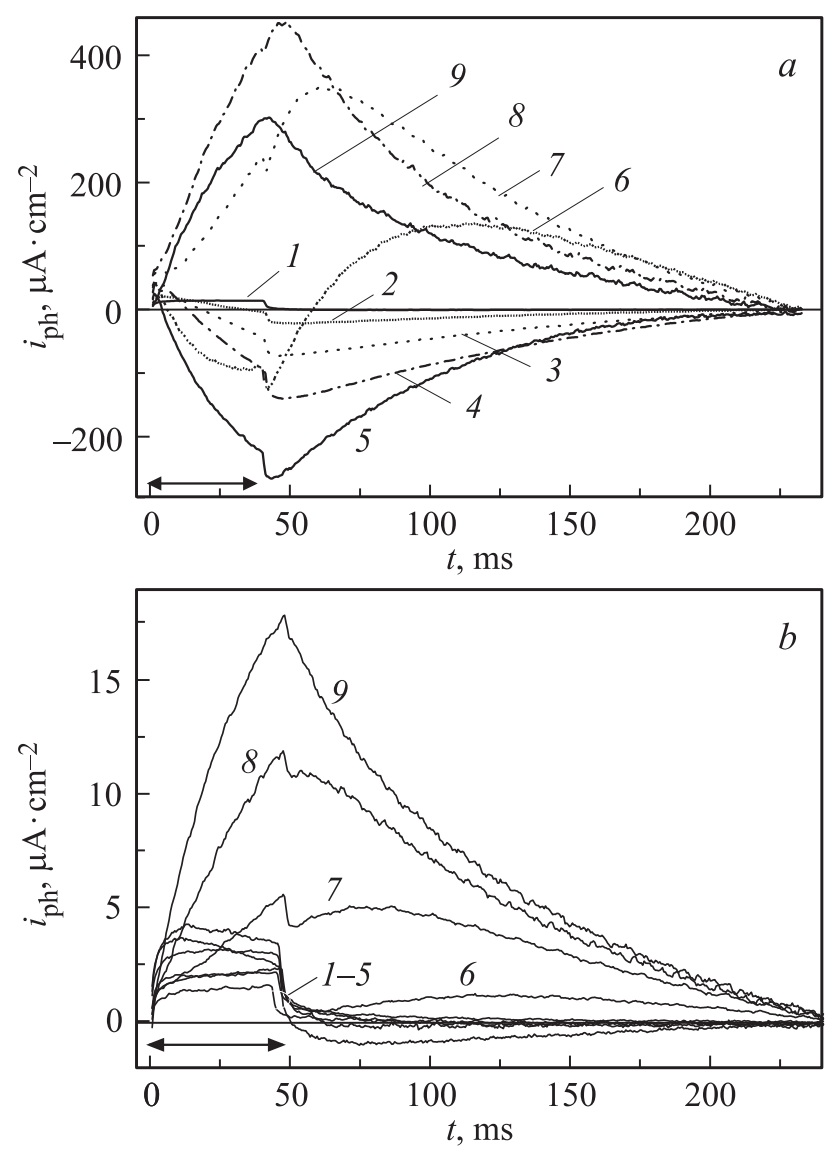

Рис. 2. Кинетика фотоотклика образца G130 без засветки (a) и при наличии промежуточной засветки (b). $T=200$ (1), 210 (2), 220 (3), 230 (4), 240 (5), 250 (6), 260 (7), 270 (8) и $280 \mathrm{~K}(9)$. Стрелкой отмечен период светового возбуждения.

pe $170 \mathrm{~K}$, типична для реакции полупроводникового кристалла на фотовозбуждение. Кинетика релаксации содержит участок быстрого спада фотоотклика (за время $\sim 2 \mathrm{~ms}$ ), сменяющийся более медленной экспоненциальной релаксацией, что можно интерпретировать как снижение концентрации неравновесных свободных носителей и термоэмиссию неравновесного заполнения дефектов соответственно. С увеличением температуры в процессе релаксации спад медленной компоненты сменяется ростом (рис. 1, $a$, кривые 2,3). Заметно сокращение начального участка быстрой релаксации, так что при $200 \mathrm{~K}$ кинетика сигнала больше напоминает фотоотклик обратносмещенного диода Шоттки или $p-n$-перехода - полупроводниковых структур, обладающих встроенным электрическим полем. Дальнейший нагрев обнаруживает в релаксации фотоотклика дополнительный участок медленного нарастания сигнала, заметный при температуре $T>200 \mathrm{~K}$ (кривая 5 на рис. 1). Аномалии фотоотклика подобного типа наблюдались и на других образцах $\mathrm{TlGaSe}_{2}$, не рассматриваемых в настоящей работе. Аномалии связаны с процессами в объеме кристалла, поскольку они регистрируются 
также при возбуждении участка поверхности образца, не граничащей с контактами.

Наличие аномалии фотоотклика зависит от предыстории возбуждения образца. На рис. 2 приведены результаты, соответствующие двум различным начальным условиям регистрации фотовозбуждения образца G130. В обоих случаях образец был предварительно отожжен (выдержан в течение часа в темноте при температуpe $350 \mathrm{~K}$ ), далее он охлаждался и проводилась регистрация реакции кристалла на фотовозбуждение в процессе медленного нагрева. На рис. 2, $b$ приведены результаты, полученные после отжига и промежуточной засветки образца при комнатной температуре в течение $10 \mathrm{~min}$ лампой накаливания $40 \mathrm{~W}$ с расстояния $5 \mathrm{~cm}$. Заметно, что засветка уменьшает фотоотклик более чем на порядок величины и значительно ослабляет аномалии кинетики. Более длительные промежуточные засветки приводили к регистрации кинетики фотоотклика обычного вида, сопоставимой с изменением проводимости образца при освещении. Аналогичная засветка предварительно отожженного образца, проведенная при температуре $78 \mathrm{~K}$, не влияла на регистрацию аномалий.

\section{4. Анализ и обсуждение данных}

4.1. Предв арительные замечания. Область температур регистрации $T \sim 170-200 \mathrm{~K}$ (кривые $1-4$ на рис. $1, a)$ для кинетики релаксации составляющей с отрицательной амплитудой (назовем ее $\beta$-компонента) хорошо сопоставима с обнаруженным в PICTS-исследованиях диапазоном температур регистрации термоэмиссии с центра $A 4(165-195 \mathrm{~K})$ [6]. Изложенное выше позволяет объяснить аномалии кинетики фотоотклика размораживанием электрета, сформированного центрами A4. Действительно, для кристалла в сегнетоэлектрическом состоянии следует предполагать перераспределение генерируемых светом неравновесных носителей заряда в электрическом поле доменной структуры, что может вести к пространственной неоднородности заполнения центров локализации заряда. В процессе нагрева такие области пространственно неоднородного заполнения центров в объеме кристалла - электреты, сформировавшиеся при низкой температуре в сегнетоэлектрической фазе, - будут оставаться замороженными вплоть до последовательной термоактивации процессов перезарядки соответствующих центров. В принципе можно предположить возможность существования и скрытых электретов, т.е. формирование системы взаимно скомпенсированных электретов. Предположение о связи аномалий кинетики фотоотклика с размораживанием электретов согласуется с зависимостью наблюдения аномалий от предыстории образца и вполне уместно для области $T \sim 170-200 \mathrm{~K}$. Появление добавочной $\beta$-компоненты при $T>200 \mathrm{~K}$ (кривая 5 на рис. 1 ) также соответствует обнаруженной ранее температуре начала регистрации термоэмиссии с центра $A 5(T=200 \mathrm{~K})$ [6]. В области
$T>200 \mathrm{~K}$ аномалии кинетики с нагревом все менее сопоставимы с фотооткликом диода. Здесь кинетика релаксации больше напоминает релаксацию фотоотклика пленок аморфных сплавов As-Se [15], хотя аномалии в нашем случае наблюдаются для монокристалла и при напряженности внешнего поля, на три порядка меньшей. Авторы [15] объясняли подобные аномалии кинетики формированием в объеме образца области пространственного заряда, что в принципе уместно и в нашем случае для области $T \sim 170-200 \mathrm{~K}$. Однако область температур регистрации добавочной $\beta$-компоненты $T \sim 201-280 \mathrm{~K}$ (кривые $5-12$ на рис. $1, b$ ) значительно шире диапазона температур наблюдения термоэмиссии с центра $A 5(200-240 \mathrm{~K})$ [6]. Отметим и следующую особенность: немонотонный характер кинетики фотоотклика несопоставим с изменением проводимости при освещении кристалла и заставляет полагать наличие в регистрируемом сигнале дополнительной фотовольтаической составляющей. В пользу такой интерпретации свидетельствует и наличие заметных начальных участков быстрых изменений сигнала (рис. $1, a$ ), характерных для фотовольтаической компоненты [16]. Эти участки сопровождают аномалии кинетики и хорошо различимы в области $T<200 \mathrm{~K}$ (рис. $1, a$ ). Однако при $T>200 \mathrm{~K}$ изменения сигнала на этих участках малы (рис. $1, b$ и $2, a)$ по сравнению с величиной аномального фотоотклика.

4.2. Анализ температурной зависимости кинетики релаксации аномального фотоо тк ли ка. Для детального анализа вариации с температурой аномального фотоотклика была использована аппроксимация кинетики релаксации модельной функцией. Аппроксимация проводилась последовательно в каждой температурной точке области $T \sim 200-300 \mathrm{~K}$ на основе специально развитого программного модуля итерационного определения параметров модели методом согласованного градиентного спуска с возможностью изменения соотношения масштабов приращений параметров в процессе подгонки [17]. Практически во всей рассмотренной области температур ${ }^{1}$ кинетика релаксации фотоотклика может быть хорошо представлена модельной функцией (алгебраической суммой нескольких экспоненциальных составляющих) вида

$$
I_{p h}(t)=C_{0}+A_{0} \exp (-\alpha t)-B_{0} \exp (-\beta t)+D_{0} \exp (-\gamma t),
$$

где $A_{0}, B_{0}, D_{0}, \alpha, \beta, \gamma>0$ и $\gamma>\alpha$. Полученные в результате анализа изменения с температурой параметров модели представлены на рис. 3. Можно отметить изменение характера зависимости от температуры показателя степени составляющей с отрицательной амплитудой $\beta$-компоненты. Значения $\beta$ малы и фактически не изменяются в области $T \sim 200-230 \mathrm{~K}$, а затем $(T>230 \mathrm{~K})$ экспоненциально растут. Различия поведения просматриваются и на зависимости от температуры амплитуды или

\footnotetext{
${ }^{1}$ Исключение составляли узкие специфические температурные участки, проблемные для использованных алгоритмов аппроксимации [18].
} 

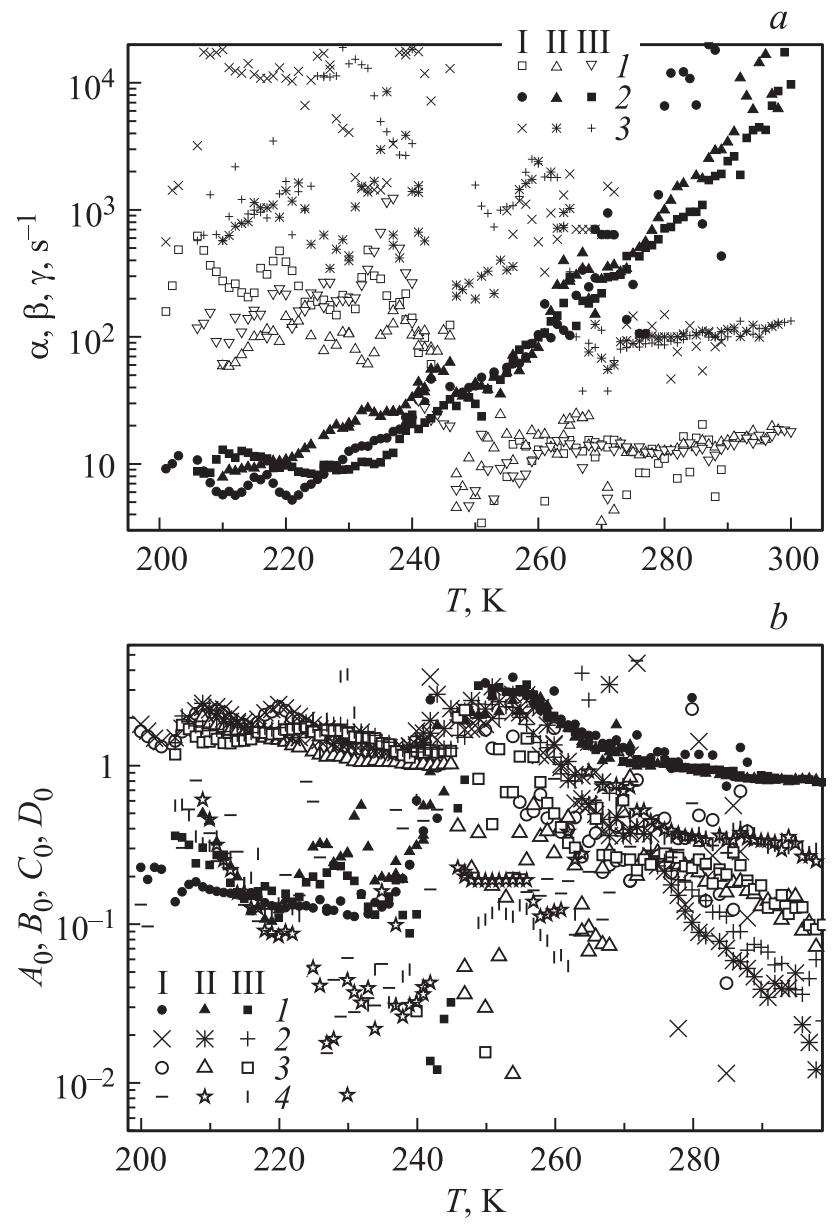

Рис. 3. Изменение с температурой показателя степени (a) и амплитуды $(b)$ экспоненциальных составляющих кинетики релаксации фотоотклика кристалла $\mathrm{TlGaSe}_{2}$ (образец Gl30). Зависимость 1 соответствует $\alpha$-компоненте, $2-\beta$-компоненте, $3-\gamma$-компоненте, $4-$ вклад постоянной составляющей. Использован набор данных, соответствующий рис. 2, $a$ (I), а также результаты анализа иных независимых испытаний, проведенных по идентичной методике регистрации данных (II и III).

вклада экспоненциальных составляющих в релаксацию (рис. $3, b)$. В области $T \sim 200-230 \mathrm{~K}$ основной вклад вносят $\beta$ - и $\gamma$-компоненты, тогда как вклад $\alpha$-компоненты на порядок меньше. Попутно отметим, что эта область температур хорошо сопоставима с температурой регистрации термоэмиссии с центра $A 5$ ( $T \sim 200-240 \mathrm{~K})$ [6], что дает основания полагать, что и в данной области температур преимущественный механизм возникновения аномалий кинетики фотоотклика связан с наличием внутреннего поля в кристалле. При дальнейшем повышении температуры наблюдается резкий рост (более чем на порядок) величины вклада $\alpha$-компоненты с переходом через максимум при $T \sim 255 \mathrm{~K}$ и доминирующим характером ее вклада в релаксацию в последующем. Здесь $(T>255 \mathrm{~K})$ вклад $\alpha$-компоненты падает до значений, близких к единице при $T \sim 300 \mathrm{~K}$, где исчезают и аномалии фотоотклика. Последнее позволяет ассоцииро- вать эту компоненту релаксации с током проводимости кристалла. Отметим, что с целью исключения влияния изменений с температурой величины фотоотклика для аппроксимации использовались нормированные зависимости $i_{n}=i(n \tau) / I_{0}$, где $I_{0}-$ разность максимального и минимального значений сигнала, определенных на всем интервале времен регистрации кинетики релаксации фотоотклика, $i_{n}-$ сигнал, зарегистрированный в $n$-й временно́й точке самплирования $(n=1-2000)$. Вместе с тем в окрестности максимума $(T \sim 255 \mathrm{~K})$ вклад $\alpha$-компоненты превышает единицу и сопоставим с наблюдающимся здесь же максимумом вклада $\beta$-компоненты (рис. 3, $b$ ). Зависимости такого типа характерны для параметрического резонанса. В пользу такой интерпретации свидетельствует и особенность приведенной кинетики образцов (рис. $1, b$ и 2, $a$ ): отсутствие выхода на стационарное значение сигнала как в режиме фотовозбуждения, так и за время релаксации фотоотклика. Таким образом, можно сделать вывод, что в области $T \sim 230-280 \mathrm{~K}$ фотоотклик кристалла формируется в динамическом режиме, имеет характер автоколебаний, вызванных возмущающим воздействием периодического возбуждения светом. Для выяснения физической природы механизма возникновения резонанса важен анализ сигнала на промежутках времени резкого изменения интенсивности фотовозбуждения [16].

4.3. Анализ начального участка релаксации фотоотклика. На рис. 4 показан результат численного дифференцирования начального участка кинетики релаксации в нескольких выбранных температурных точках. Заметно значительное изменение $\Delta i_{n}$ на относительно коротком начальном интервале времени. На данном интервале в регистрируемом сигнале можно ожидать присутствие вклада фотовольтаической составляющей, тогда как последующее развитие процесса релаксации фотоотклика в принципе может быть связано

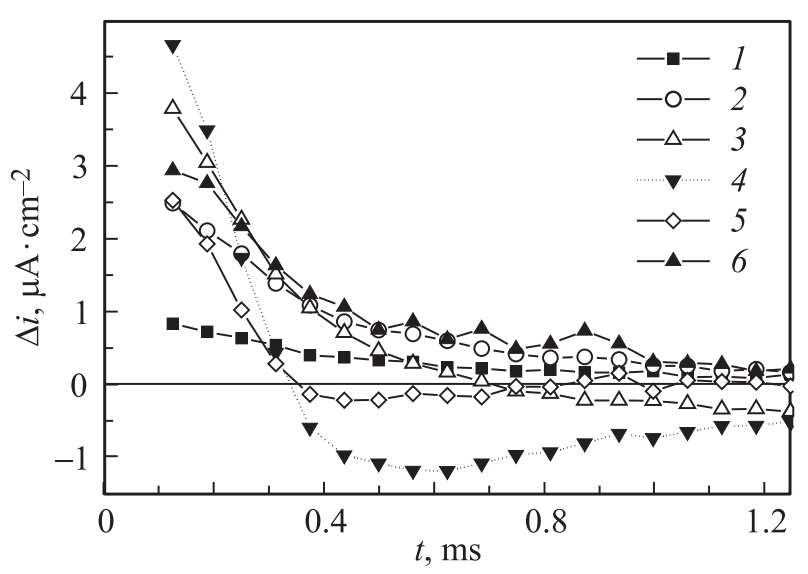

Рис. 4. Результат численного дифференцирования кинетики релаксации фотоотклика $\mathrm{TlGaSe}_{2}$ (образец $\left.\mathrm{Gl30}\right) . T=170$ (1), 190 (2), 200 (3), 210 (4), 220 (5) и $250 \mathrm{~K}$ (6). Использован набор данных, соответствующий зависимостям, приведенным рис. $1, b$. 
исключительно с токами проводимости и экранирования. Следует заметить, что при ширине входной щели монохроматора $4 \mathrm{~mm}$ лезвие модулятора, расположенного непосредственно перед щелью, перекрывает световой поток за время, соответствующее приблизительно десяти точкам самплирования. Однако вследствие наличия задержки в системе синхронизации регистрации данных на рисунке можно выделить начальный участок в пятьшесть точек самплирования (кривые $1-3$ ), где изменения $\Delta i_{n}$ соответствуют фактически линейной зависимости, сопоставимой с линейным спадом аппаратной функции регистрации заднего фронта светового импульса. На рис. 4 заметно, что наклон начального участка быстрого спада фотоотклика изменяется с температурой, что в принципе согласуется с приведенным выше предположением о размораживании электретов в областях температур $T \sim 170-200$ и $200-230 \mathrm{~K}$. Вместе с тем на рисунке можно заметить и некоторую выпуклость начального участка приведенных зависимостей, явно выраженную для кривых 4-6. Заметная выпуклость кривых 4-6 характерна для начального момента развития процесса релаксации, т. е. доминирует при высоких уровнях фотовозбуждения. Опираясь на это, аномалии кинетики фотоотклика кристалла можно объяснить вторичным явлением, сопутствующим регистрации фотовольтаических токов. Действительно, за конечный период фотовозбуждения под воздействием фотовольтаической эдс происходит определенное перераспределение свободных носителей заряда, генерируемых при освещении кристалла. В процессе релаксации возбужденного состояния следует ожидать принципиально различных ситуаций для случая, когда фотовольтаический ток обусловлен наличием внутреннего нескомпенсированного поля в кристалле (электрета), и случая фотовольтаического тока иной природы, не связанной с наличием внутреннего поля [19]. С резким уменьшением интенсивности фотовозбуждения релаксация пространственной неоднородности свободных носителей, сформировавшейся под действием фотоэдс, в первом случае будет происходить в условиях воздействия все того же внутреннего поля. Во втором случае такого поля нет, а следовательно, уже на ранних этапах релаксации следует ожидать значительной величины соответствующего тока экранирования со знаком, обратным знаку фотовольтаического тока. Чтобы подчеркнуть различие такой фотовольтаический ток называют еще фотогальваническим. Именно фотогальванический ток на интервалах времени резкого изменения светового потока может вести к эффекту занижения величины регистрируемого фотоотклика, поскольку соответствующий ему ток экранирования течет внутри кристалла и не вносит вклада в сумму токов, регистрируемых во внешней измерительной цепи. При относительно небольшой подвижности носителей заряда [20] пространственная неоднородность распределения свободных носителей в объеме кристалла, формируемая под действием фотогальванической эдс, вполне может вести к возникновению параметрического резонанса токов проводимости и экранирования.

\section{5. Заключение}

В результате изучения изменения с температурой кинетики аномального фотоотклика $\mathrm{TlGaSe}_{2}$ предложены механизмы формирования фотоотклика кристалла. Аномалии, наблюдающиеся в области $T \sim 170-200 \mathrm{~K}$, а также в области $T \sim 200-230 \mathrm{~K}$ можно интерпретировать как обусловленные пространственной неоднородностью заполнения центров локализации заряда. В более высокотемпературной области $T \sim 230-280 \mathrm{~K}$ аномалии фотоотклика формируются вследствие параметрического резонанса, возникающего в условиях периодического возбуждения кристалла светом с $h v \geq E_{g}$ и приложенного к кристаллу внешнего поля. Обнаружено, что при световом возбуждении кристалла изменение проводимости сопровождается дополнительным эффектом возникновения фотогальванического тока. Учитывая доминирующий характер этого явления в условиях фотовозбуждения, можно предположить, что и формирование электретов может быть следствием наличия фотогальванического тока.

Фотогальванические явления, обнаруженные еще в [21], в настоящее время исследуются в рамках практических приложений, связанных с записью голографической информации, где важным фактором является увеличение разрешающей способности среды, определяющей плотность записи. В таких приложениях структурирование электрических характеристик объема кристалла интересно в микронном масштабе. В нашем случае фотогальванический эффект, наблюдающийся на макроуровне, обнаружен в пределах всего образца, что может иметь и ряд иных практических приложений. Тем более что при выбранной частоте фотовозбуждения максимум проявления эффекта наблюдается при $\sim 255 \mathrm{~K}$, что недалеко от комнатной температуры и относительно легко технически достижимо.

\section{Список литературы}

[1] S. Johnsen, Z. Liu, J.A. Peters, J.-H. Song, S. Nguyen, C.D. Malliakas, H. Jin, A.J. Freeman, B.W. Wessels, M.C. Kanatzidis. J. Am. Chem. Soc. 133, 10030 (2011).

[2] J.A. Kalomiros, N. Kalkan, M. Hanias, A.N. Anagnostopoulos, K. Kambas. Solid State Commun. 96, 601 (1995).

[3] S. Ozdemir, M. Bucurgat. Solid State Sci. 33, 25 (2014).

[4] V. Grivickas, A. Odrinski, V. Bikbajevas, K. Gulbinas. Phys. Status Solidi B 250, 160 (2013).

[5] А.П. Одринский. ФТТ 56, 331 (2014).

[6] M.H. Seyidov, F.A Mikailzade, T. Uzun, A.P. Odrinsky, E. Yakar, V.B. Aliyeva, S.S. Babayev, T.G. Mammadov. Physica B 483, 82 (2016).

[7] А.А. Волков, Ю.Г. Гончаров, Г.В. Козлов, С.П. Лебедев, А.М. Прохоров, Р.А. Алиев, К.Р. Аллахвердиев. Письма в ЖЭТФ 37, 517 (1983). 
[8] Н.Т. Мамедов, Е.С. Крупников, А.М. Панич. ФТТ 31, 290 (1989).

[9] A.M. Panich, D.C. Ailion, S. Kashida, N. Gasanly. Phys. Rev. B 69, 245319 (2004).

[10] Г.И. Абуталыбов, Л.С. Ларионкина, Н.А. Рагимова. ФТТ 31, 312 (1989).

[11] K.R. Allakhverdiev, M.A. Aldzanov, T.G. Mamedov, E.Yu Salaev. Solid State Commun. 58, 295 (1986).

[12] M.A. Aldzhanov, N.G. Guseinov, Z.N. Mamedov. Phys. Status Solidi A 100, K145 (1987).

[13] Н.А. Абдуллаев, Т.Г. Мамедов, Р.А. Сулейманов. ФНТ 27, 915 (2001).

[14] Ch. Hurter, M. Boilou, A. Mitonneau, D. Bois. Appl. Phys. Lett. 32, 821 (1978)

[15] A. Česnys, G. Juška, E. Montrimas. In: Semiconducting chalcogenide glass II properties of chalcogenide glasses / Eds R. Fairman, B. Ushkov. Ser. Semiconductors and semimetals. V. 79. Elsevier, Academic Press, London (2004). Ch. 2. P. $15-55$.

[16] А.П. Одринский, М.-H.Yu. Seyidov, R.A. Suleymanov, Т.Г. Мамедов, В.Б. Алиева. ФТТ 58, 696 (2016).

[17] А.П. Одринский. ФТП 49, 294 (2015).

[18] A.A. Istratov, O.F. Vyvenko. Rev. Sci. Instrum. 70, 2, 1233 (1999).

[19] В.И. Белинчер, Б.И. Стурман. УФН 130, 415 (1980).

[20] A.F. Qasrawi, N.M. Gasanly. Mater. Res. Bull. 39, 1353 (2004).

[21] A.M. Glaas, D. von der Linde, T.J. Negran. Appl. Phys. Lett. 25, 15 (1974). 